\title{
A critical role for STIM1 in filopodial calcium entry and axon guidance
}

Sangwoo Shim ${ }^{1,2,3,4}$, James Q Zheng ${ }^{1,2^{*}}$ and Guo-li Ming ${ }^{3,4,5}$

\begin{abstract}
Background: Stromal interaction molecule 1 (STIM1), a $\mathrm{Ca}^{2+}$ sensor in the endoplasmic reticulum, regulates store-operated $\mathrm{Ca}^{2+}$ entry (SOCE) that is essential for $\mathrm{Ca}^{2+}$ homeostasis in many types of cells. However, if and how STIM1 and SOCE function in nerve growth cones during axon guidance remains to be elucidated.

Results: We report that STIM1 and transient receptor potential channel 1 (TRPC1)-dependent SOCE operates in Xenopus spinal growth cones to regulate $\mathrm{Ca}^{2+}$ signaling and guidance responses. We found that STIM1 works together with TRPC1 to mediate SOCE within growth cones and filopodia. In particular, STIM1/TRPC1-dependent SOCE was found to mediate oscillatory filopodial $\mathrm{Ca}^{2+}$ transients in the growth cone. Disruption of STIM1 function abolished filopodial $\mathrm{Ca}^{2+}$ transients and impaired $\mathrm{Ca}^{2+}$-dependent attractive responses of Xenopus growth cones to netrin-1. Finally, interference with STIM1 function was found to disrupt midline axon guidance of commissural interneurons in the developing Xenopus spinal cord in vivo.
\end{abstract}

Conclusions: Our data demonstrate that STIM1/TRPC1-dependent SOCE plays an essential role in generating spatiotemporal $\mathrm{Ca}^{2+}$ signals that mediate guidance responses of nerve growth cones.

Keywords: Axon guidance, STIM1, SOCE, TRPC1, Calcium, Netrin-1, Filopodial $\mathrm{Ca}^{2+}$ entry, $\mathrm{Ca}^{2+}$ oscillation, Calcium homeostasis

\section{Background}

Guided axonal growth and regeneration depend on the motile growth cone at the tip of axons to extend and navigate through a complex environment to reach specific targets for neuronal connections. It is well established that the nerve growth cone needs to maintain an optimal range of intracellular $\mathrm{Ca}^{2+}$ concentration $\left(\left[\mathrm{Ca}^{2+}\right]_{\mathrm{i}}\right)$ for its motility and responses to extracellular cues [1]. The cytoplasmic $\mathrm{Ca}^{2+}$ homeostasis is regulated by $\mathrm{Ca}^{2+}$ entry from the extracellular environment, internal $\mathrm{Ca}^{2+}$ release and replenishment of the intracellular store [2,3]. However, how neuronal growth cones coordinate guidance cue-induced $\mathrm{Ca}^{2+}$ influx, internal $\mathrm{Ca}^{2+}$ release and $\mathrm{Ca}^{2+}$ store replenishment to maintain proper guidance behaviors is unknown. Store-operated $\mathrm{Ca}^{2+}$ entry (SOCE) was originally characterized in non-excitable cells as an indispensable

\footnotetext{
* Correspondence: james.zheng@emory.edu

'Departments of Cell Biology and Neurology, Emory University School of Medicine, Atlanta, GA 30078, USA

${ }^{2}$ Center for Neurodegenerative Diseases, Emory University School of

Medicine, Atlanta, GA 30322, USA

Full list of author information is available at the end of the article
}

$\mathrm{Ca}^{2+}$ influx mechanism to replenish internal stores [2,3]. It is triggered by $\mathrm{Ca}^{2+}$ depletion from ER through the ER $\mathrm{Ca}^{2+}$ sensor protein, stromal interacting molecule 1 (STIM1). In response to $\mathrm{Ca}^{2+}$ depletion, STIM1 oligomerizes and translocates to ER and plasma membrane junctions, where it interacts with and activates storeoperated $\mathrm{Ca}^{2+}$ (SOC) channels that include TRPC1 and Orail proteins $[2,3]$.

In the nervous system, SOCE has been seen to exist in a number of cell types [4-7] and implicated in synaptic plasticity, axon branching, neuropathic pain and fly motor circuit function [6-10]. However, the existence of SOCE and STIM1, and their potential contribution to the intracellular $\mathrm{Ca}^{2+}$ homeostasis and signaling in axon guidance is not well established. Axonal growth cones are highlighted by two types of actin-based motile membrane protrusions, filopodia and lamellipodia [11]. Of these two structures, lamellipodia are considered to be responsible for growth cone locomotion, whereas filopodia are believed to function in sensing of the environment during axon pathfinding [11-13]. Interestingly, rapid $\mathrm{Ca}^{2+}$ transients in growth cone filopodia have been shown to be 
involved in growth cone responses to extracellular cues $[14,15]$. But how $\mathrm{Ca}^{2+}$ signals are generated in filopodia and whether SOCE is involved in this process remain unknown. Here we report that SOCE operates in Xenopus spinal growth cones and depends on STIM1 and TRPC1. Importantly, we find that SOCE mediates spontaneous and netrin-1-potentiated filopodial $\mathrm{Ca}^{2+}$ entries within growth cones. We further provide evidence that STIM1and TRPC1-dependent SOCE is required for attractive guidance responses of growth cones to netrin-1. Finally, we show that STIM1 is required for midline axon guidance of commissural interneurons in the developing Xenopus spinal cord in vivo. Our data suggest that SOCE is an essential component of intracellular $\mathrm{Ca}^{2+}$ homeostasis and signaling that regulate neuronal growth cone guidance.

\section{Results}

\section{Cloning and expression of Xenopus STIM1}

We first cloned Xenopus STIM1 (XSTIM1; 668 a.a.), which exhibited $72.8 \%$ identity to human STIM1 (685 a.a.; Additional file 1: Figure S1). Whole-mount in situ hybridization of developing Xenopus embryos showed that STIM1 is highly expressed in the dorsal part of the developing embryo, including the neural tube at stages when active axon guidance occurs (Figure 1A, top panels). Coronal sections of Xenopus embryos confirmed the expression of STIM1 mRNA in the neural tube, as well as in the notochord and somites (Figure 1A, bottom panels). RTPCR analysis from dissected tissues further confirmed that XSTIM1 mRNA is expressed in neural tube and notochord of early tailbud Xenopus embryos (Figure 1B). Immunofluorescence analysis using anti-STIM1 antibody and fluorescent phalloidin to stain F-actin showed that STIM1 protein is ubiquitously distributed in Xenopus spinal neuron, including soma, neurites and growth cones (Figure 1C). Robust expression of XSTIM1 throughout the growth cone and in filopodia is better seen at higher magnifications (Figure 1D). These results show that Xenopus STIM1 is expressed in developing neural tissues and neuronal growth cones of developing axons.

\section{STIM1- and TRPC1-dependent SOCE in Xenopus neuronal growth cones}

To study the function of STIM1 in growth cones, we generated wild-type (XSTIM1-WT) and two mutant forms of XSTIM1: a dominant negative form (DN, XSTIM1-D65A$\triangle \mathrm{CT}$ ) and a constitutively active form (CA, XSTIM1-CT) (Figure 2A) based on previous studies of mammalian STIM1 mutants [16]. We first examined whether STIM1dependent SOCE operates in neuronal growth cones by $\mathrm{Ca}^{2+}$ imaging analysis using fluo-4 calcium indicator. Xenopus neurons were first bathed in $\mathrm{Ca}^{2+}$-free media with cyclopiazonic acid (CPA; $25 \mu \mathrm{M})$, an endoplasmic reticulum $\mathrm{Ca}^{2+}$ pump inhibitor, to deplete intracellular
$\mathrm{Ca}^{2+}$ stores. Subsequent addition of extracellular $\mathrm{Ca}^{2+}$ induced a marked and rapid rise in the intracellular $\mathrm{Ca}^{2+}$ concentration $\left(\left[\mathrm{Ca}^{2+}\right]_{\mathrm{i}}\right)$ in the growth cone (Figure $2 \mathrm{~B}$ and $\mathrm{C}$ ), suggesting the presence of functional SOCE in neuronal growth cones [4-7]. Importantly, we found that the SOCE was largely abolished in growth cones expressing either dominant negative Xenopus STIM1 (XSTIM1-DN, Figure $2 \mathrm{~B}$ and $2 \mathrm{C}$ ) or dominant negative human STIM1 (hSTIM1-DN) (Figure 2C), indicating that the elevation in $\left[\mathrm{Ca}^{2+}\right]_{\mathrm{i}}$ requires the function of STIM1. In addition, knock-down of TRPC1 channels by morpholino oligonucleotides against XTRPC1 (XTRPC1-MO) abolished SOCE (Figure 2D). Therefore, TRPC1 is also an essential component of store-operated $\mathrm{Ca}^{2+}$ (SOC) channel complex as suggested by previous studies in saliva gland cells [17]. These results show that store-operated $\mathrm{Ca}^{2+}$ entry operates in neuronal growth cones and requires STIM1 and TRPC1.

\section{STIM1-dependent SOCE mediates netrin-1-induced $\mathrm{Ca}^{2+}$ elevation in growth cones}

Netrin-1 is known to guide axonal growth cone through a $\mathrm{Ca}^{2+}$-dependent pathway $[1,18]$ and netrin-1-induced increase in $\left[\mathrm{Ca}^{2+}\right]_{\mathrm{i}}$ in growth cones requires both intracellular $\mathrm{Ca}^{2+}$ release and $\mathrm{Ca}^{2+}$ influx through channels on the plasma membrane [19-21]. Consistent with previous studies [21-23], bath application of netrin-1 (10 ng/ml final concentration) induced a significant rise in $\left[\mathrm{Ca}^{2+}\right]_{\mathrm{i}}$ in Xenopus growth cones (Figure 2E). Importantly, overexpression of XSTIM1-DN abolished the sustained $\mathrm{Ca}^{2+}$ elevation within neuronal growth cones in response to netrin-1 (Figure 2E). Previous studies from ours and others have shown that TRPC1 is required for netrin-1induced sustained $\mathrm{Ca}^{2+}$ elevation [21,23]. We have observed an association of TRPC1 with STIM1 in Xenopus brain lysates (Additional file 2: Figure S2), similar to what has been shown in mammalian cells $[2,16,24]$. Therefore, netrin-1 may activate STIM1-dependent SOCE through TRPC1 in neuronal growth cones.

STIM1-dependent SOCE generates filopodial $\mathrm{Ca}^{2+}$ entries in Xenopus neuronal growth cones

Localized $\mathrm{Ca}^{2+}$ transients in filopodia play an important role in filopodial motility and growth cone responses to extracellular cues [14]. We thus examined the potential role for SOCE in $\mathrm{Ca}^{2+}$ entry in growth cone filopodia. Here we used a membrane tethered calcium indicator Lck-GCaMP3 [25] to reliably monitor $\mathrm{Ca}^{2+}$ influx through the plasma membrane. Using a similar protocol as in Figure 2B-D, we measured both the incidence and frequency of fast $\mathrm{Ca}^{2+}$ entries in filopodia by high-speed (30 frames/sec) wide-field fluorescent imaging. We found that store $\mathrm{Ca}^{2+}$ depletion and re-addition of external $\mathrm{Ca}^{2+}$ experiment led to $\mathrm{Ca}^{2+}$ entries in about $25 \%$ filopodia 
(Figure 3; Additional file 3: Movie 1). High frequency time-lapse traces of the integrated intensity of the LckGCaMP3 fluorescence and a kymograph representation clearly demonstrated that filopodial $\mathrm{Ca}^{2+}$ entries are independent of growth cone $\mathrm{Ca}^{2+}$ transients (Figure 3A-C; Additional file 3: Movie 1). Importantly, both the incidence and frequency of these filopodial $\mathrm{Ca}^{2+}$ entries were largely abolished by inhibition of XSTIM1 with XSTIM1DN overexpression (Figure 3D and E; Additional file 4: Movie 2) or TRPC1 knockdown by XTRPC1-MO (Figure 3D and E; Additional file 5: Movie 3). These results thus indicate that STIM1- and TRPC1-dependent SOCE is indispensable for filopodial $\mathrm{Ca}^{2+}$ entries within neuronal growth cones.
Previous studies have revealed that Xenopus growth cones exhibit spontaneous filopodial $\mathrm{Ca}^{2+}$ transients that are closely associated with growth cone motility $[14,15,26]$. Using Lck-GCaMP3, we also observed robust periodic, spontaneous calcium entries in filopodia of Xenopus growth cones in a Modified Ringer solution that contains $1 \mathrm{mM}$ extracellular $\mathrm{Ca}^{2+}$ (Figure 4A; Additional file 6: Movie 4). Importantly, both the incidence and frequency of the entries were also substantially reduced by inhibition of STIM1 and TRPC1 function (Figure 4B and C; Additional file 7: Movie 5 and Additional file 8: Movie 6), suggesting that STIM1/TRPC1-dependent SOCE is involved in generating and maintaining oscillatory patterns of spontaneous filopodial $\mathrm{Ca}^{2+}$ transients.
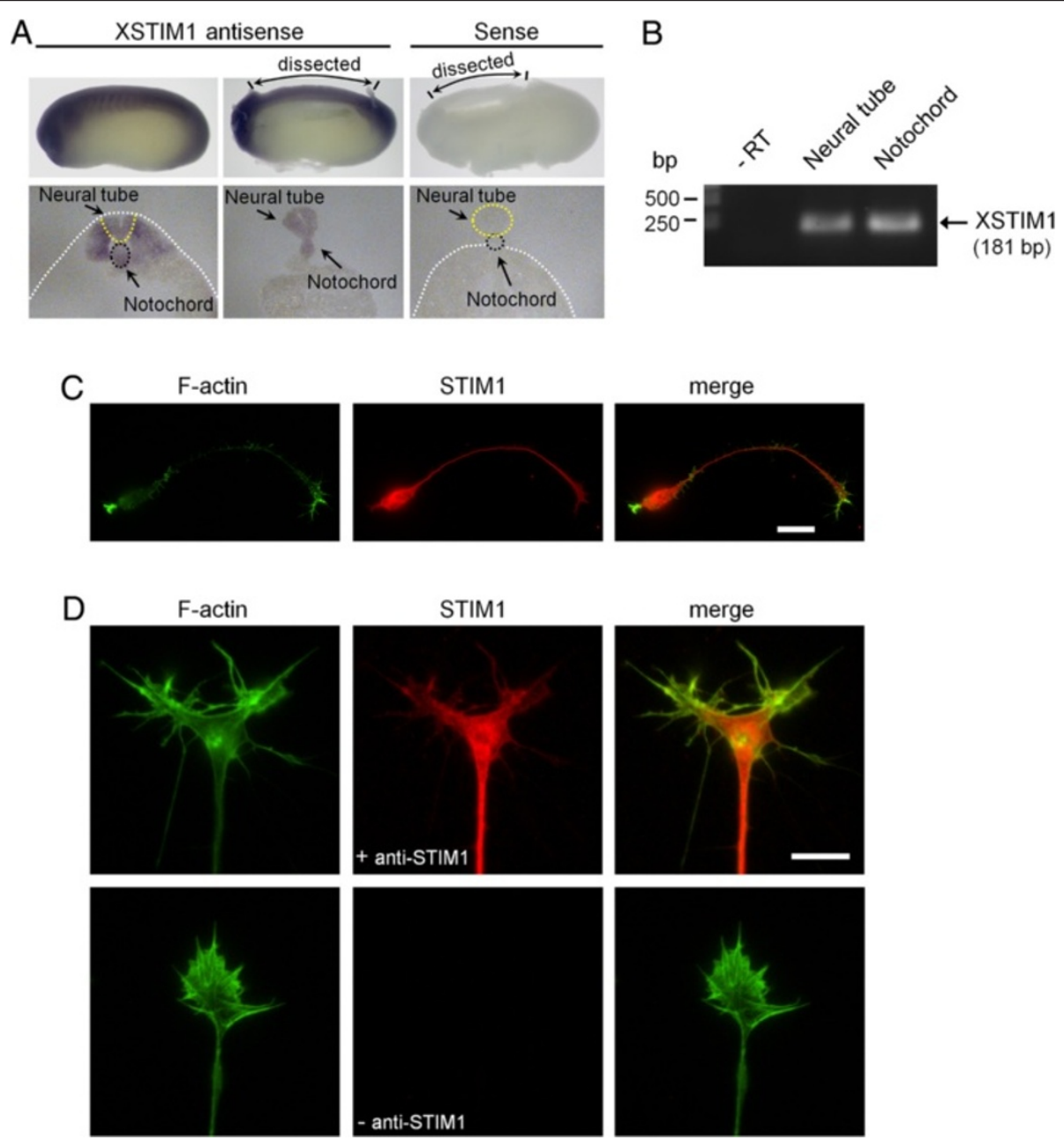

Figure 1 Xenopus STIM1 is expressed in developing neural tissues and neuronal growth cones. (A) Sample images of whole-mount (top) and cross-section (bottom) in situ hybridization analysis of the mRNA expression of XSTIM1 in developing Xenopus embryos. Left, antisense; right, sense probe. Dotted lines delineate the boundaries of neural tube and notochord. (B) RT-PCR detection of XSTIM1 mRNA from RNA samples extracted from state 25-26 Xenopus neural tube and notochord tissues. -RT lane is the negative control of the RT-PCR on neural tube tissue RNA in the absence of a reverese transcriptase. (C) Representative immunofluorescence images of cultured Xenopus spinal neurons labeled for STIM1 (red) and F-actin (phalloidin: green). Scale bar: 20 Mm. (D) Representative immunofluorescence images of growth cones labeled for STIM1 (red) and F-actin (green). Negative control processed without STIM1 antibody (without STIM1, bottom) shows absence of immunolabeling. Scale bar: $10 \mu \mathrm{m}$. 
Bath application of netrin-1 (10 ng/ml final concentration) was found to potentiate both the incidence and frequency of filopodial $\mathrm{Ca}^{2+}$ entries of Xenopus spinal growth cones (Figure 4D-F; Additional file 9: Movie 7), consistent with previous study using Fluo-4 [15]. We found that this increase in filopodial $\mathrm{Ca}^{2+}$ entries by netrin-1 was abolished when STIM1 function was inhi- bited by XSTIM1-DN (Figure 4E and F). Over-expression of morpholino against XTRPC1 (XTRPC1-MO) also compromised the potentiation of filopodial $\mathrm{Ca}^{2+}$ entries by netrin-1 (Figure 4E and F). Therefore, STIM1/TRPC1dependent SOCE mediates the netrin-1-dependent potentiation of oscillatory filopodial $\mathrm{Ca}^{2+}$ entries in neuronal growth cones.
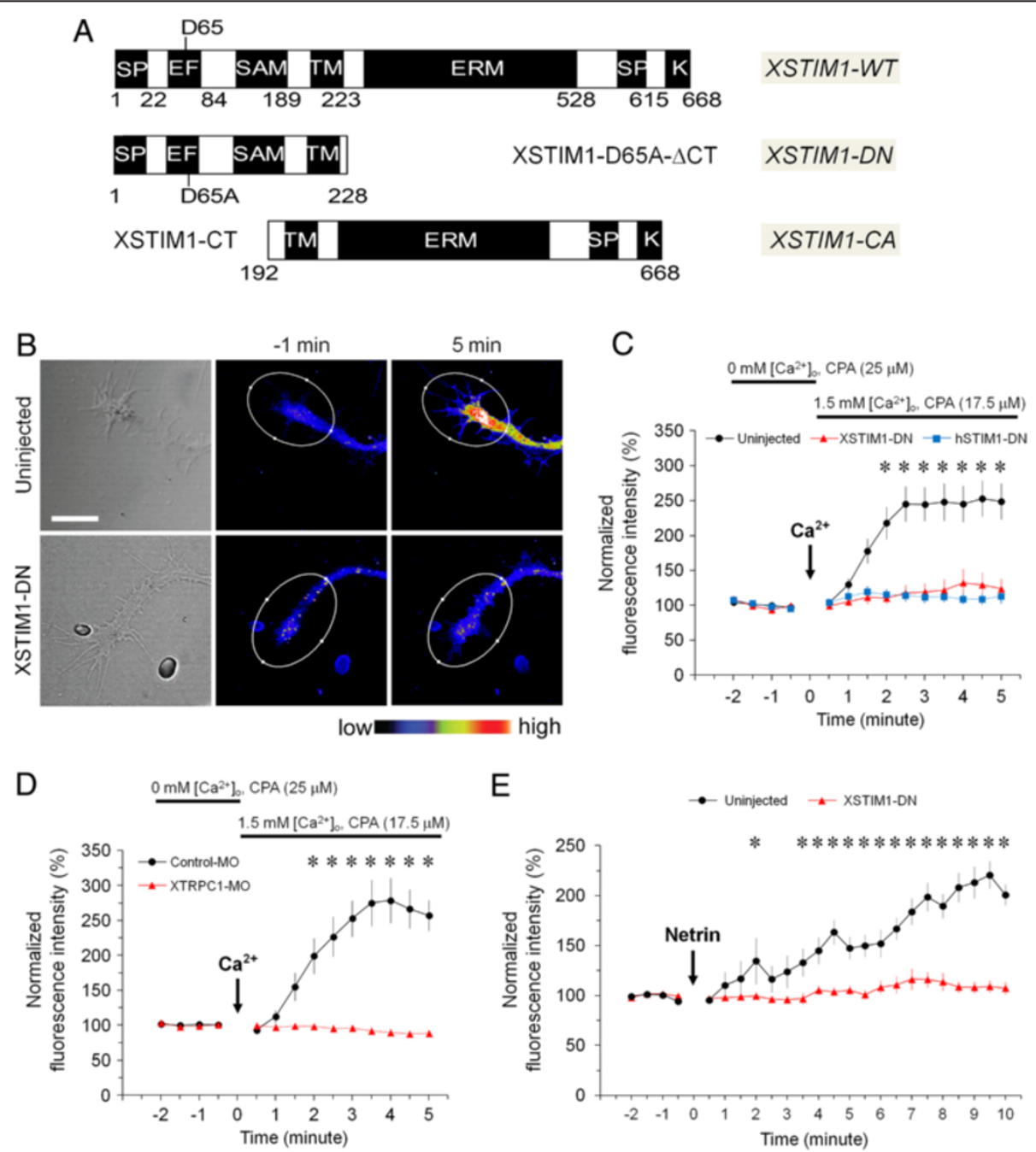

Figure 2 STIM1-dependent SOCE operates and mediates netrin-1-induced $\mathrm{Ca}^{2+}$ elevation in Xenopus neuronal growth cones. (A) A schematic diagram of full-length wild-type (WT) and mutant constructs of XSTIM1. (B) Bright field and pseudocolor images of fluo-4 fluorescence of growth cones of Xenopus spinal neurons from the uninjected or mcherry-XSTIM1-DN injected embryos in the presence of CPA in Ca ${ }^{2+}$-free media, before and after the re-addition of $1.5 \mathrm{mM} \mathrm{Ca}^{2+}$ bath solution. Pseudocolors indicate $\mathrm{Ca}^{2+}$ levels, with white as the highest and black as the lowest. Scale bar: $10 \mu \mathrm{m}$. (C) Summary of internal $\mathrm{Ca}^{2+}$ store depletion-induced $\mathrm{Ca}^{2+}$ entry in growth cones at different time points before and after re-addition of $1.5 \mathrm{mM} \mathrm{Ca}^{2+}$. The fluorescence intensity was normalized to the average fluorescence intensity of 2 min baseline levels prior to $\mathrm{Ca}^{2+}$ re-addition. Values represent mean \pm s.e.m. $\left(\mathrm{n}=25\right.$ for control, $\mathrm{n}=10$ for XSTIM1-DN and $\mathrm{n}=19$ for hSTIM1-DN; ${ }^{*}$ indicates $P<0.01$; Bootstrap-test). (D) XTRPC1 is required for store depletion-evoked $\mathrm{Ca}^{2+}$ entry in neuronal growth cones. Summary of internal $\mathrm{Ca}^{2+}$ store depletion-induced $\mathrm{Ca}^{2+}$ entry in growth cones from the control-MO or XTRPC1-MO injected embryos at different time points before and after the re-addition of $1.5 \mathrm{mM} \mathrm{Ca}^{2+}$. Values represent mean \pm s.e.m. $(n=12$ for control, and $n=13$ for XTRPC1-MO; * indicates $P<0.01$; Bootstrap-test). (E) XSTIM1 is required for netrin-1-induced $\mathrm{Ca}^{2+}$ elevation in growth cone. Summary of time course of $\mathrm{Ca}^{2+}$ changes in neuronal growth cones from uninjected or mCherry-XSTIM1-DN mRNA injected embryos. The fluorescence intensity was normalized to the average fluorescence intensity of 2 min baseline levels prior to the netrin-1 application $(10 \mathrm{ng} / \mathrm{ml})$. Values represent mean \pm s.e.m. $(n=6$ for control and $n=9$ for XSTIM1-DN; * indicates $P<0.05$; Bootstrap-test) 

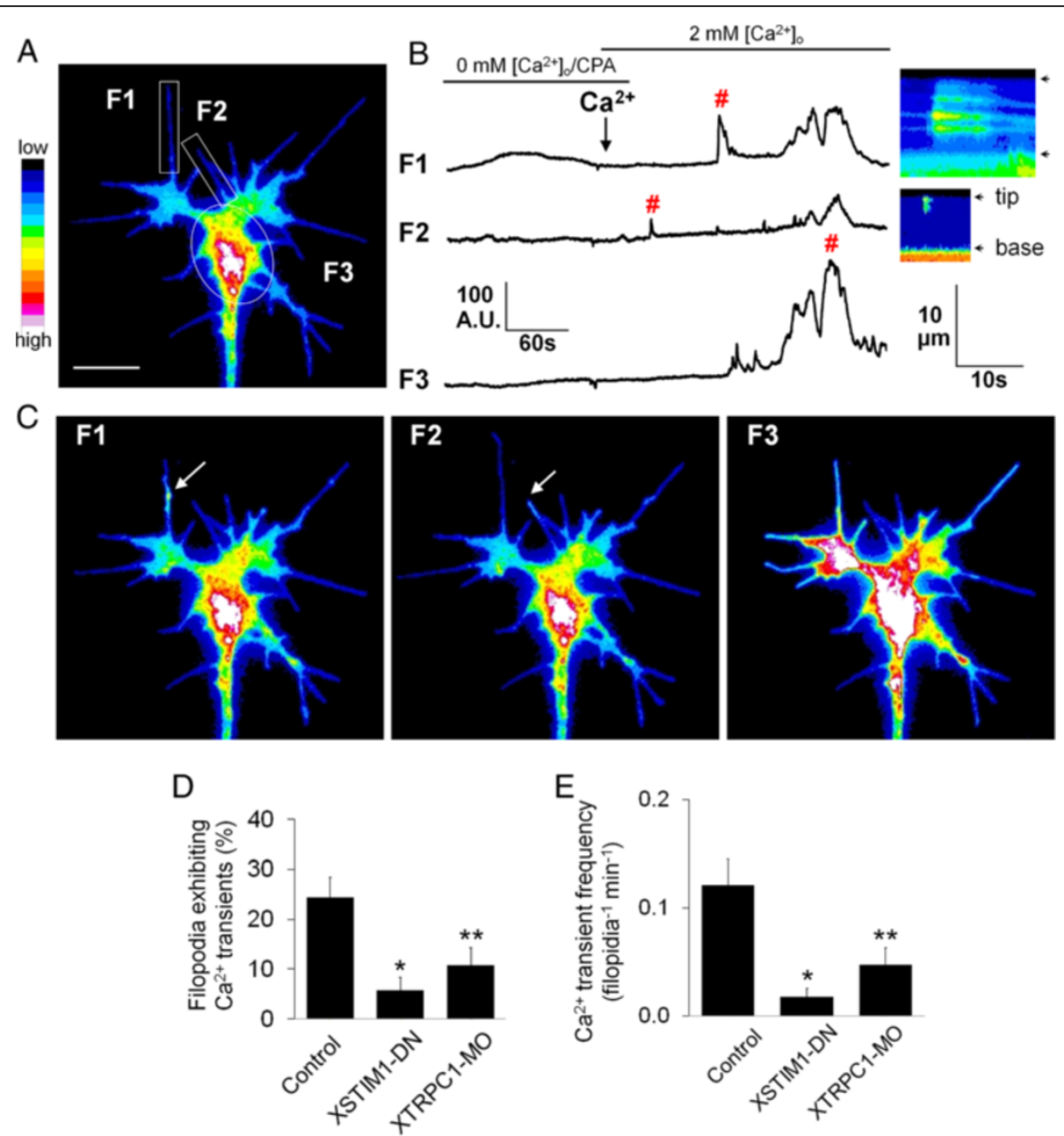

Figure 3 STIM1-dependent SOCE generates filopodial $\mathrm{Ca}^{2+}$ entries in Xenopus neuronal growth cones. (A) A pseudocolored Lck-GCaMP3 fluorescent $\mathrm{Ca}^{2+}$ image of growth cone showing rectangular ROls (region of interest) used to measure fluorescent intensities over time. Pseudocolors indicate $\mathrm{Ca}^{2+}$ levels, with white as the highest and black as the lowest. Scale bar, $10 \mu \mathrm{m}$. (B) Representative traces of Lck-GCaMP3 fluorescent $\mathrm{Ca}^{2+}$ signals profile measured in two filopodia (F1, F2) and a growth center (F3) over 7 min period of store-depletion and re-addition of extracellular $\mathrm{Ca}^{2+}$. Images were captured at 200 milliseconds intervals. \#, indicates filopodial and global $\mathrm{Ca}^{2+}$ transients that are shown in (C). Right images are kymographs generated from a segmented line along the filopodia from the tip to the base using NIH ImageJ. The arrowheads denote tip and base of filopodia. (C) Representative pseudocolored Lck-GCaMP3 fluorescent $\mathrm{Ca}^{2+}$ images at the time point as indicated by \# in B. The arrows show the initiation of filopodial $\mathrm{Ca}^{2+}$ transients. (D-E) The incidence (D) and frequency (E) of filopodial $\mathrm{Ca}^{2+}$ transients were determined in control $(n=21)$, XSTIM1-DN $(n=12)$, XTRPC1-MO $(n=10)$ expressing filopodia. ${ }^{*} P<0.005$ and ${ }^{* *} p<0.05$ compared with control condition using t-test. Values represent mean \pm s.e.m.

The membrane tethered calcium indicator lck-GCaMP3 also provides an opportunity to map the entry sites of $\mathrm{Ca}^{2+}$ in filopodia. We thus analyzed the sites of initial filopodial $\mathrm{Ca}^{2+}$ entry in Xenopus growth cones by kymography analysis. We found that, although the initial sites of $\mathrm{Ca}^{2+}$ entry distributed throughout the length of a filopodium, a large portion of the $\mathrm{Ca}^{2+}$ entry sites (42-59\%) were found at the filopodial tip (Figure 4G). When filopodial $\mathrm{Ca}^{2+}$ entries under different conditions (store-operated, spontaneous, and netrin-1-induced) were examined, no difference was seen on the location of $\mathrm{Ca}^{2+}$ entry sites in filopodia (Figure 4G). Therefore, the tip of the filopodia appears to be the primary site of SOCE-mediated $\mathrm{Ca}^{2+}$ entry in nerve growth cones.

STIM1 proteins reside predominantly in the ER, and undergo rapid and reversible translocation into ER-plasma membrane junctions to interact with and activate SOC channels following store depletion in non-excitable cells [27]. Live cell imaging of Xenopus neurons expressing YFP tagged STIM1 showed that YFP-XSTIM1-WT appeared to translocate into filopodia after store $\mathrm{Ca}^{2+}$ depletion, as revealed by pseudocolored images and phase overlay images with mCherry (Figure 5; Additional file 10: Movie 8). Together with the presence of STIM1 in Xenopus growth 


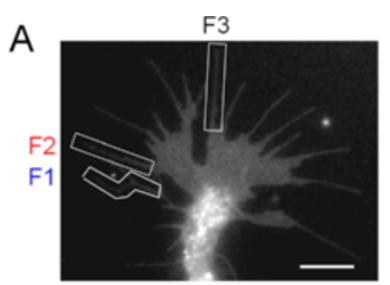

B
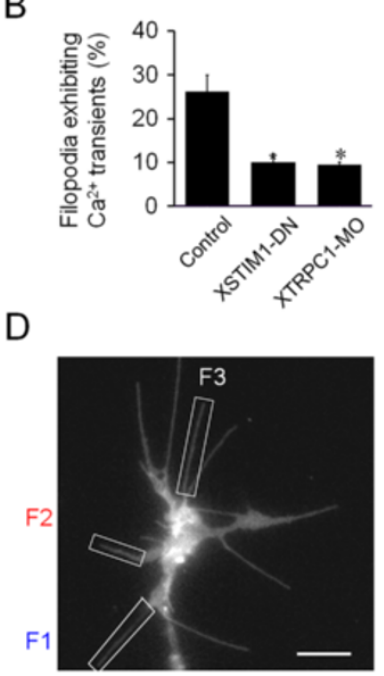

E

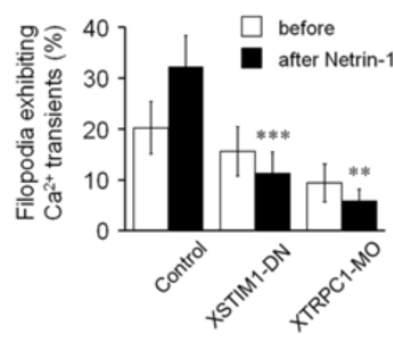

A.U.

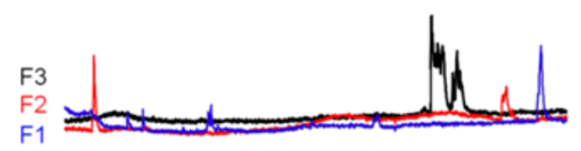

C

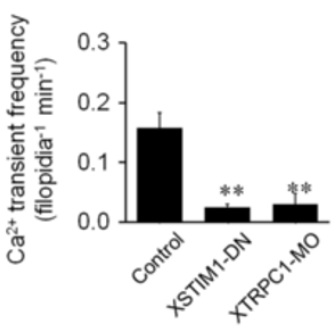

A.U. $\frac{40}{605}$

Netrin-1

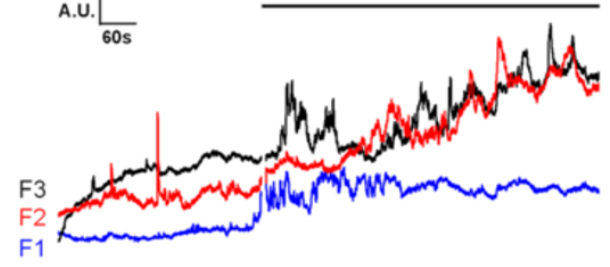

$\mathrm{F}$

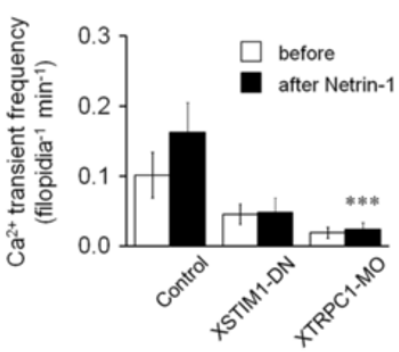

G

$80 \quad(\%)$
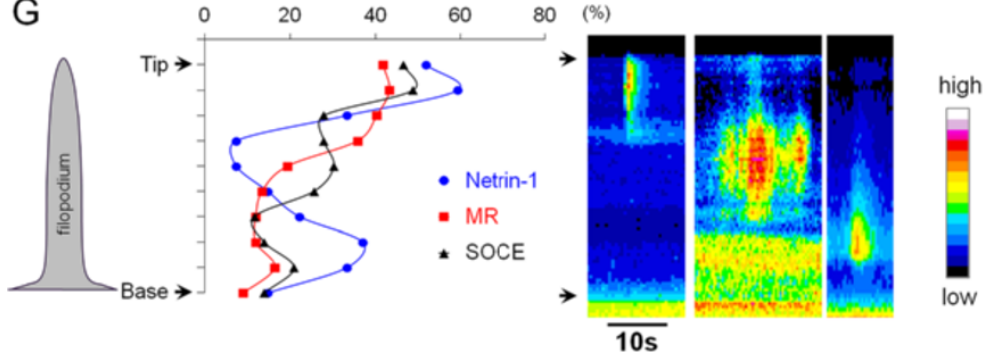

Figure 4 STIM1/TRPC1-dependent SOCE mediates the spontaneous and netrin-1-potentiated filopodial $\mathrm{Ca}^{2+}$ entries. (A) Left panel; a Lck-GCaMP3 fluorescent $\mathrm{Ca}^{2+}$ image of a Xenopus spinal growth cone showing three ROIs (F1, F2 and F3) encompassing the filopodia used to measure fluorescent intensities over time (right panels). Scale bar, $10 \mu \mathrm{m}$. The incidence (B) and the frequency (C) of the spontaneous filopodial $\mathrm{Ca}^{2+}$ transients were significantly attenuated by XSTIM1-DN $(n=30)$ or XTRPC1-MO $(n=30)$, when compared to the control $(n=34 ; * P<0.001$ and ${ }^{* *} \mathrm{p}<0.005$, Student's t-test). Values represent mean \pm s.e.m. (D) A Lck-GCaMP3 fluorescent $\mathrm{Ca}^{2+}$ image of a growth cone showing three ROIs (left panel) and their representative traces of $\mathrm{Ca}^{2+}$ signals in three filopodia (F1, F2, F3) before and after bath application of netrin-1 (10 ng/ml) (right panel) in the presence of Sp-CAMP $(25 \mu \mathrm{M})$. Scale bar, $10 \mu \mathrm{m}$. (E-F) Netrin-1 potentiated the incidence (E) and the frequency (F) of filopodial $\mathrm{Ca}^{2+}$ transients in spinal growth cones (control; $n=14$ ) and this potentiation was abolished by XSTIM1-DN $(n=8)$ and XTRPC1-MO $(n=10)$. *P $<0.005$ and ${ }^{* * *} \mathrm{p}<0.05$ (Student's t-test). Values represent mean \pm s.e.m. (G) Filopodia tips are the major site of initiation of filopodial $\mathrm{Ca}^{2+}$ entry as revealed by kymographs of $\mathrm{Ca}^{2+}$ signals in filopodia using Lck-GCaMP3 in modified Ringers saline (MR; $\left.n=67\right)$, netrin-1 exposure $(n=27)$ and $\mathrm{Ca}^{2+}$ re-addition after depletion (SOCE; $n=43$ ). The $y$ axis represents the path distance along the filopodia divided into 10 portions and the $x$ axis represents time. The arrows denote the tip and base of filopodia. 
cones and their filopodia by immunostaining (Figure 1D), these results suggest an intriguing possibility that STIM1 proteins may become spatially reorganized into growth cone filopodia after activation by store-depletion to further activate SOC channels that may include TRPC1.

\section{XSTIM1 is required for growth cone guidance}

To test whether STIM1-dependent SOCE is required for growth cone guidance in response to netrin-1, we employed a well-established in vitro growth cone turning assay $[19,20,23,28]$. Previous studies have shown that netrin1 , a classical guidance cue, induces growth cone turning responses that are mediated by $\mathrm{Ca}^{2+}$ from both extracellular and intracellular sources [19-21,29]. In a microscopic gradient of netrin-1 $(5 \mu \mathrm{g} / \mathrm{ml}$ in the pipette, $\sim 5 \mathrm{ng} / \mathrm{ml}$ reaching the growth cone), Xenopus growth cones of overnight culture (12-20 hrs) without laminin coating exhibited robust chemoattractive turning within 30 minutes (Figure 6A). Importantly, expression of XSTIM1-DN or XSTIM1-CA in Xenopus spinal neurons completely abolished netrin-1-induced attraction, and interestingly resulted in repulsion (Figure 6A and B). Expression of wild-type STIM1 (WT) produced no effect on netrin1 -induced attractive turning (Figure 6A and B). Overexpression of the dominant negative human STIM1 (hSTIM1-DN) [16] also eliminated netrin-1-induced attraction and converted it to repulsion (Figure 6B). The neurite extension rate in a netrin-1 gradient was not significantly different under these conditions [29], except XSTIM1-CA which slightly reduced the growth rate (Figure 6C). Thus, proper function of XSTIM1 is essential for netrin-1-induced growth cone turning responses of Xenopus spinal neurons in vitro. Together with the previous studies showing that TRPC1 knockdown abolished the netrin-1-induced attractive growth cone turning responses $[20,21]$, the results indicate that STIM1/TRPC1-dependent SOCE may play a critical role in growth cone guidance.

To assess whether STIM1 is required for axon guidance in vivo, we examined the midline axon guidance of commissural interneurons in the developing Xenopus spinal cord, which is known to require netrin-1 signaling [20,23]. Commissural interneuron axons in developing Xenopus embryos were specifically identified with the 3A10 monoclonal antibody [20]. In stage 25-26 embryos, 3A10positive commissural axons extend toward and across the midline in a highly organized manner (Figure 7A and B). In contrast, a significant percentage of commissural axons derived from YFP-XSTIM1-DN injected embryos wondered around and failed to cross the midline with some even went out of the spinal cord (Figure 7C). For simplicity, we scored both types of guidance defects as the one not crossing and presented and quantified the percentage of crossed axons (Figure 7F). XSTIM1-DN markedly reduced the percentage of crossed axons to about $50 \%$ of the control groups (uninjected and GFP injection only)

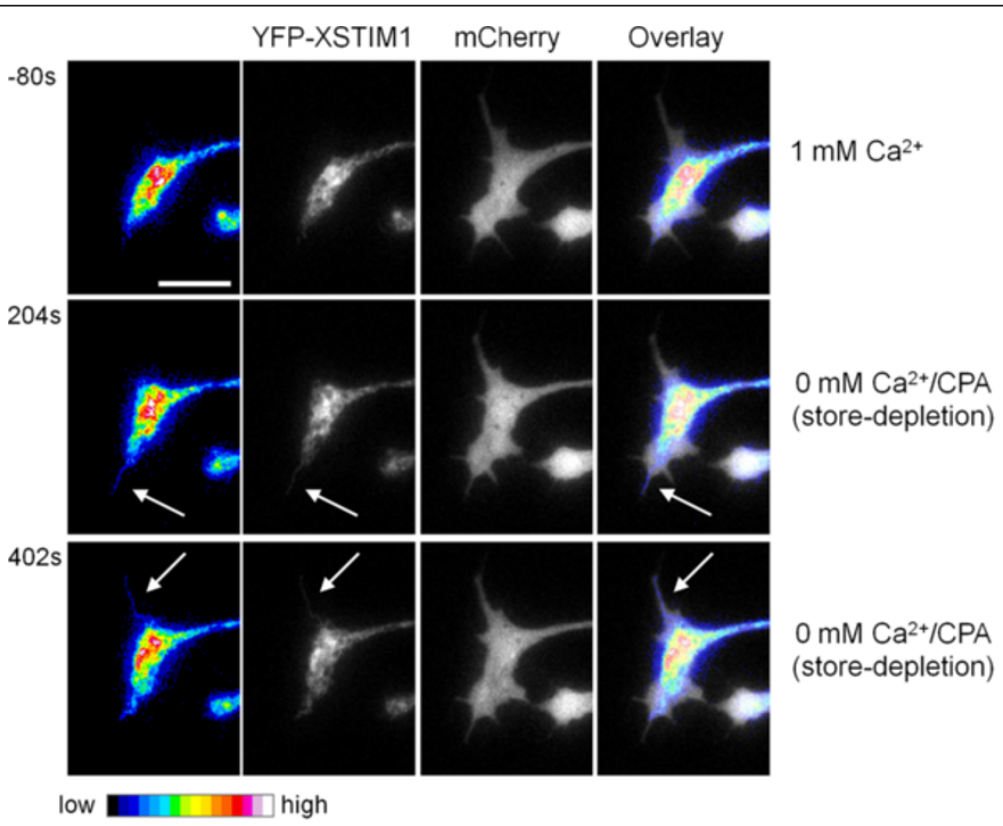

Figure 5 Dynamic translocation of STIM1 into neuronal filopodia in response to store-depletion. Representative time-lapse fluorescent images of growth cone expressing YFP-XSTIM1 before $\left(1 \mathrm{mM} \mathrm{Ca}^{2+}\right)$ and after store $\mathrm{Ca}^{2+}$ depletion $\left(0 \mathrm{mM} \mathrm{Ca}{ }^{2+} / \mathrm{CPA}\right)$. mCherry was co-expressed to mark filopodia and growth cone. Images were pseudocolored to enhance the observation of intensity changes. The arrows indicate newly translocated XSTIM1 proteins into neuronal filopodia. Scale bars: $10 \mu \mathrm{m}$. 
A

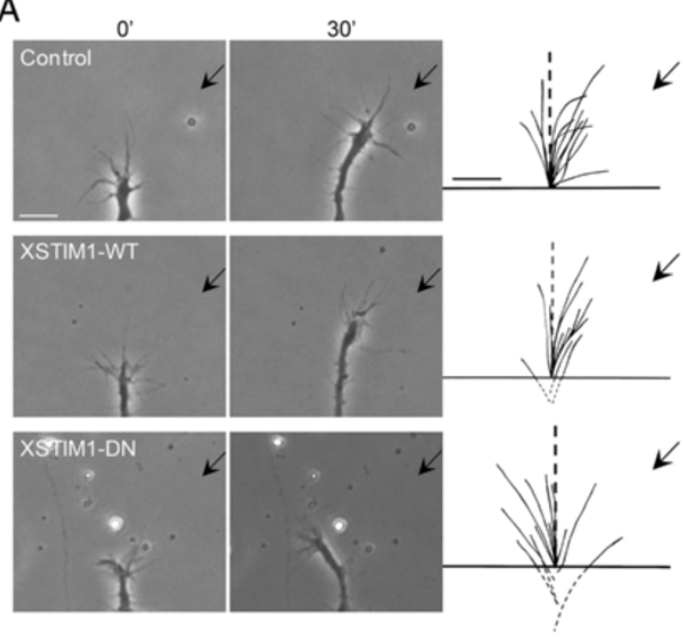

B
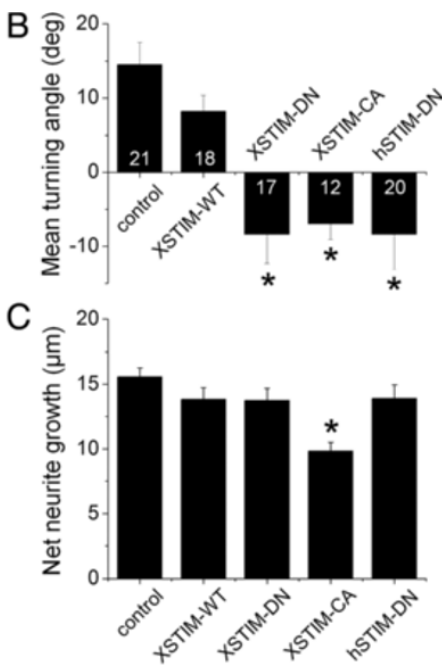

Figure 6 XSTIM1 is required for attractive turning responses of neuronal growth cones to a netrin-1 gradient. (A) Sample images of growth cone turning responses in a gradient of netrin-1 of a control Xenopus spinal neuron, and neurons derived from embryos injected with mRNA encoding wild-type (YFP-XSTIM1-WT), or dominant negative mutant (YFP-XSTIM1-DN). The left two columns of images show neuronal growth cones at the start $(0 \mathrm{~min})$ and the end of exposure $(30 \mathrm{~min})$ to a netrin-1 gradient ( $5 \mathrm{\mu g} / \mathrm{ml}$ in the pipette). The right column shows superimposed trajectories of neurite extension during the 30' period for a sample population of 12 neurons under the each condition. The origin is the center of the growth cone and the original direction of growth is vertical. Arrows indicate the direction of the gradient. Scale bars: $10 \mu \mathrm{m}$. (B) Summary of mean turning angles of growth cones in responses to a gradient of netrin-1 under different conditions. The number associated with the bar graph indicates the number of growth cones analyzed. Values represent mean \pm s.e.m. (* indicates $P<0.01$; Bootstrap-test). (C) Summary of net neurite growth during the 30 minutes turning assay under different conditions. Values represent mean \pm s.e.m. $\left({ }^{*}\right.$ indicates $P<0.05$; Bootstrap-test).

and the WT group. Similarly, over-expression of YFPXSTIM-CA, but not YFP-XSTIM1-WT, also led to significant midline guidance defects (Figure 7D and F). On the other hand, none of these molecular manipulations significantly affected the numbers of commissural neurons in the developing Xenopus spinal cord (Figure 7G). Taken together, these results demonstrated that STIM1 is requried for the midline axon guidance of commissural interneurons in the developing Xenopus spinal cord in vivo.

\section{Discussion}

$\mathrm{Ca}^{2+}$ is a key second messenger that mediates a wide range of neuronal activity and responses to extracellular stimuli [1]. Spatiotemporally-restricted $\mathrm{Ca}^{2+}$ signals can steer growth cones in responses to extracellular guidance cues and are thought to be generated from $\mathrm{Ca}^{2+}$ influx through the plasma membrane as well as $\mathrm{Ca}^{2+}$ release from internal store $[1,18]$. However, how these two events are coupled to sustain $\mathrm{Ca}^{2+}$ signaling during guidance responses remain unclear. SOCE is believed to be a part of the intracellular $\mathrm{Ca}^{2+}$ homeostasis machinery that maintains $\left[\mathrm{Ca}^{2+}\right]_{\mathrm{i}}$ for various neuronal functions, including optimal growth cone motility. The function of SOCE is well established in non-excitable cells and considered as a $\mathrm{Ca}^{2+}$ entry mechanism for refilling intracellular $\mathrm{Ca}^{2+}$ stores. The molecular components of SOCE include STIM, Orai, and TRP channels, which have also been identified and characterized mostly in non-excitable cells [2,3]. More recently, several studies support the presence and functional implication of SOCE in the nervous system [4-9], but the molecular composition and functional role of SOCE and STIM1 in neuronal growth cone guidance is not well established. Our data demonstrate that STIM1- and TRPC1-dependent SOCE not only operates in neuronal growth cones but also mediates filopodial $\mathrm{Ca}^{2+}$ transients and growth cone guidance both in vitro and vivo [20,30,31]. Our results are consistent with a recent report showing that STIM1 and Orai, two components of SOCE, are involved in growth cone responses to brain derived neurotrophic factor and Semaphorin-3a [32]. Importantly, our study has further identified that TRPC1 is also an essential component of SOCE and a major site of SOCE-mediated $\mathrm{Ca}^{2+}$ entry is in the filopodia, especially at the tip of the filopodia. Moreover, we have presented in vivo evidence that STIM1 is required for proper guidance of commissural axons in developing spinal cord, a classic example of netrin-1-dependent long-range growth cone guidance $[20,30,31]$. Together, the current study has clearly established a role for SOCE, involving STIM1 and TRPC1, in mediating filopodial $\mathrm{Ca}^{2+}$ entries underlying axonal growth cone guidance.

At this moment, the precise mechanism by which STIM1 and SOCE are involved in netrin-1-induced $\mathrm{Ca}^{2+}$ 

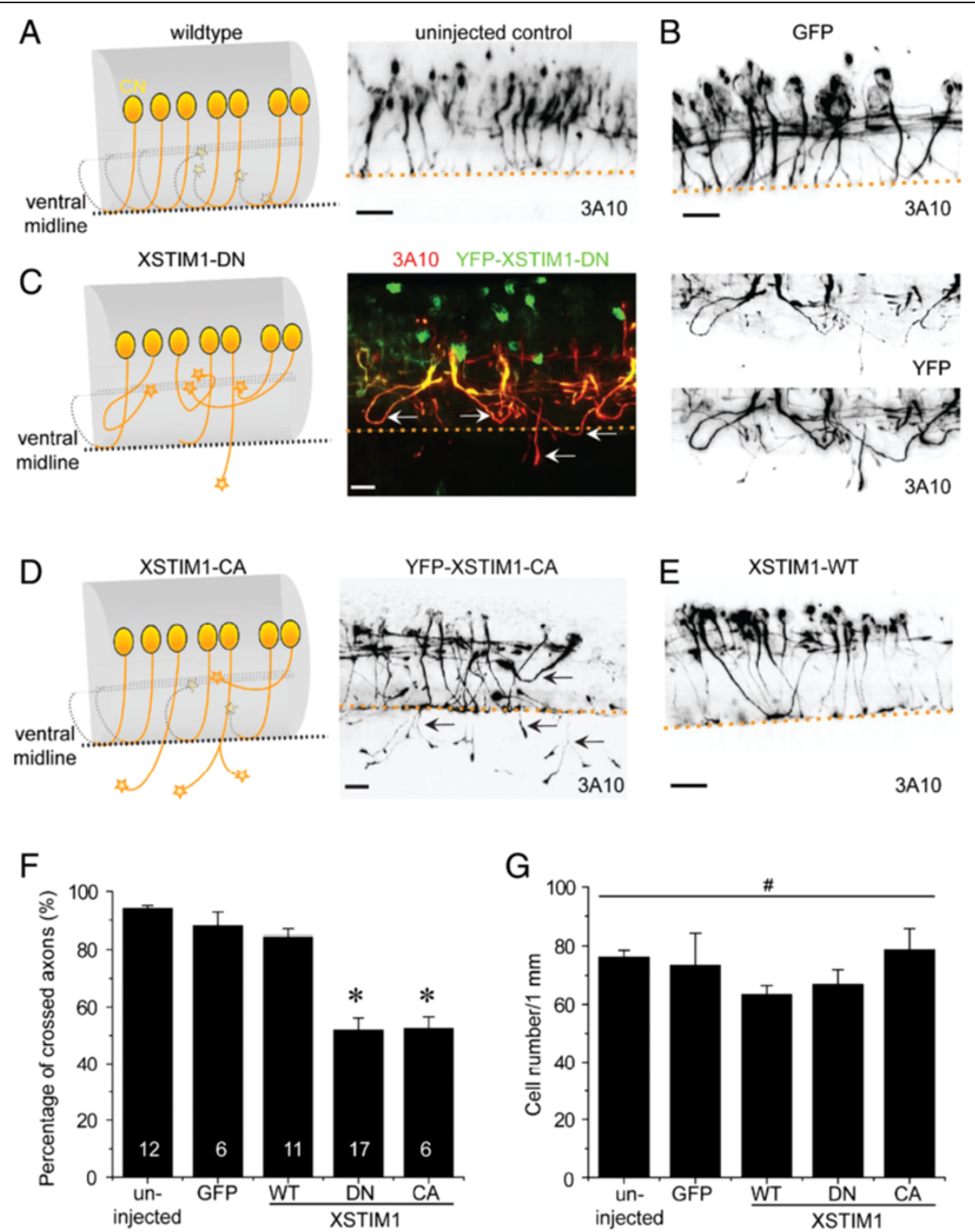

Figure 7 XSTIM1 is required for midline axon guidance of commissural interneurons in the developing Xenopus spinal cord. (A-E) Sample images of the sagittal view of commissural interneurons and their axonal projections in the Xenopus spinal cord from stage 25-26 embryos. Shown are schematic diagrams and confocal z-stack projection images of 3A10 staining of commissural interneuron axons from uninjected embryos, or embryos injected with mRNAs for GFP (B), YFP-XSTIM1-DN (C), YFP-XSTIM1-CA (D), or YFP-XSTIM1-WT (E), at the two to four cell-stage to manipulate a sub-population of neurons. Dotted line represents ventral midline; arrows point to mis-targeted axons. Scale bars: $20 \mu \mathrm{m}$. (F) Quantification of the percentage of $3 \mathrm{~A} \mathrm{O}^{+}$commissural interneurons with normal midline crossing under different experimental conditions. The number associated with the bar graph indicates the number of embryos examined under each condition. Values represent mean \pm s.e.m. ( ${ }^{*}$ indicates $P<0.01$; Bootstrap-test). (G) Summary of the density of $3 \mathrm{~A}_{1} 0^{+}$commissural neurons under each condition. The same embryos as in (F) were examined. Values represent mean \pm s.e.m. ( ${ }^{\#}$ indicates $P>0.1$; Bootstrap-test).

signaling for growth cone attraction remains unclear. Netrin-1 is known to elicit $\mathrm{Ca}^{2+}$ signals by $\mathrm{Ca}^{2+}$ influx through TRPC1 and $\mathrm{Ca}^{2+}$ release from the internal stores to induce growth cone attraction $[1,18-20]$ but the underlying mechanism remains unclear. It has been shown that brain derived neurotrophic factor (BDNF) triggers $\mathrm{Ca}^{2+}$ release from internal stores through activation of the PLC- $\gamma$ and $\mathrm{IP}_{3}$ pathway, which in turn induces SOCE through STIM1-TRPC activation [18,32-34]. Netrin-1 is considered to be in the same group of $\mathrm{Ca}^{2+}$-mediated guidance cues with BDNF. Given that PLC- $\gamma$ and $\mathrm{IP}_{3}$-induced $\mathrm{Ca}^{2+}$ release are involved in growth cone extension and navigation [22,34-36], we propose that netrin-1 may initiate intracellular $\mathrm{Ca}^{2+}$ release through activation of netrin-1 receptor Deleted in Colorectal Cancer (DCC), $\mathrm{PLC}-\gamma$ and $\mathrm{IP}_{3}$ production, which further triggers store- 
depletion, STIM1 activation, and $\mathrm{Ca}^{2+}$ influx through TRPC1 for replenishing ER $\mathrm{Ca}^{2+}$. This notion is further supported by the findings that both netrin-1 and BDNF activate PLC- $\gamma$ and Phosphatidylinositol 4,5-bisphosphate $\left(\mathrm{PIP}_{2}\right)$ hydrolysis in neurite elongation $[37,38]$. Therefore, our results provide additional evidence for the conserved signaling pathways among $\mathrm{Ca}^{2+}$-mediated guidance cues and between netrin-1 and neurotrophins.

The role of TRPC channels as SOC has been controversial, but multiple lines of evidences support TRPC as a strong candidate component of store-operated $\mathrm{Ca}^{2+}$ channels. For example, TRPC1 has been shown to be bound and activated by STIM1 and contribute to SOCE in some cells $[16,17,39-41]$. We found that STIM1 interacts with TRPC1 in embryonic neural tissues (Additional file 2: Figure S2) and that TRPC1 knockdown inhibits STIM1-mediated SOCE within growth cones and filopodia (Figures 2D, 3D and 3E), suggesting that TRPC1 is an essential component of SOCE. As STIM1 is also required for netrin-1-induced $\mathrm{Ca}^{2+}$ elevation and growth cone attraction which was shown to be mediated by TRPC1, our data support a role for STIM1 in activating TRPC1. However, we cannot rule out the possibility that STIM1 may affect $\mathrm{Ca}^{2+}$ signaling and growth cone guidance by other mechanisms, such as its effects on cAMP signaling or ER remodeling [42,43]. Recent studies also showed biochemical assembly of STIM1-TRPCs-Orai complex and functional connections between TRPC channels and Orai1 [41,44,45]. STIM1-Orai1 co-localization in response to $\mathrm{Ca}^{2+}$ depletion was reported in neuronal growth cones [32]. Therefore, it is possible that Orai also plays a role in netrin-1 signaling and guidance.

It should be noted that Lck-GCaMP3 was successfully used in distinguishing the $\mathrm{Ca}^{2+}$ signals from membrane entry from internal release from the stores $[25,46]$. Our data with Lck-GCaMP3 showing the presence of filopodial $\mathrm{Ca}^{2+}$ transients and its potentiation by netrin- 1 is consistent with the previous reports using Fluo-4 [14,15,26]. However, when compared with previous studies using Fluo-4, the incidence and frequency of filopodial $\mathrm{Ca}^{2+}$ transients observed in our study appear to be lower than those seen in the previous reports. The difference may be attributed to two possibilities. First, Lck-GCaMP3 detects $\mathrm{Ca}^{2+}$ entry events only at near-plasma membrane regions. However, fluo- 4 could detect cytosol $\mathrm{Ca}^{2+}$ changes from other sources such as intracellular stores, which will likely be missed by Lck-GCaMP3. In this regard, Lck-GCaMP3 fluorescence $\mathrm{Ca}^{2+}$ signals may be better called "filopodial $\mathrm{Ca}^{2+}$ entries" rather than filopodial $\mathrm{Ca}^{2+}$ transients. Second, we did not count the $\mathrm{Ca}^{2+}$ transients propagated from the growth cone proper and only counted the $\mathrm{Ca}^{2+}$ entry events generated within the filopodium independently of $\mathrm{Ca}^{2+}$ transients from the growth cone proper. Therefore, our data do not contradict the previous work.
It is of interest to see that the initial site of filopodial $\mathrm{Ca}^{2+}$ entry is largely localized to the tip of filopodia. Filopodia are considered to be the sensory apparatus for growth cones as they extend farther distance to detect the environmental signals. Therefore, it makes sense to have the sensory molecules accumulated at the tip for signal transduction initiation. However, the lack of quality antibodies prevented us from convincingly detecting the localization of STIM1/TRPC1 and other SOCE components at the filopodial tip. On the other hand, it has been reported that several receptors such as integrins, TRPC1 and DCC $[14,26,47]$ and signaling molecules such as Src, PAK, PKA $[48,49]$ are enriched at the tip of filopodia along with many other cytoskeleton regulatory molecules $[11,50,51]$. Therefore, it is conceivable that STIM1 and TRPC1 could function at the filopodial tip as an effective way to sense the environment and initiate $\mathrm{Ca}^{2+}$ signaling during growth cone guidance.

We report fast, highly localized and periodic spontaneous filopodial $\mathrm{Ca}^{2+}$ entries initiated independently of growth cone $\mathrm{Ca}^{2+}$ transients, which was consistent with the previous reports of oscillatory pattern of spontaneous $\mathrm{Ca}^{2+}$ transients within growth cone and filopodia during axonal growth $[14,15,52]$. The critical role for TRPC1 in generation of filopodial $\mathrm{Ca}^{2+}$ entry and its potentiation by netrin-1 is also consistent with the previous reports $[14,15,26]$. A further unexpected result was that STIM1DN mutant blocked not only the SOCE-induced filopodial $\mathrm{Ca}^{2+}$ entries that depend on STIM1 but also spontaneous and netrin-1-potentiated oscillatory filopodial $\mathrm{Ca}^{2+}$ entries, suggesting that STIM1-dependent SOCE mediates the generation and maintenance of filopodial $\mathrm{Ca}^{2+}$ entries. Thus, our visualization of oscillatory patterns of spontaneous filopodial $\mathrm{Ca}^{2+}$ entries and their inhibition by STIM1- or TRPC1-knockdown is the first demonstration of a role of STIM/TRPC1-dependent SOCE in regulating $\mathrm{Ca}^{2+}$ oscillation in neurons, which is consistent with previous findings in other cell types [53-55]. It is plausible that store $\mathrm{Ca}^{2+}$ release, transient drop in ER $\mathrm{Ca}^{2+}$, and $\mathrm{Ca}^{2+}$ entry through TRPC1 triggered by transient STIM1 activation may underlie the $\mathrm{Ca}^{2+}$ oscillations seen in growth cone filopodia. Thus, considering the functional correlation between the frequency of filopodial $\mathrm{Ca}^{2+}$ transients and growth cone outgrowth and turning [14,26], disruption of STIM1 or TRPC1 function is likely to result in the breakup of $\mathrm{Ca}^{2+}$ cycling for oscillations and subsequent attenuated frequency of filopodial $\mathrm{Ca}^{2+}$ entries, which may further cause the suppression of growth cone guidance in response to netrin-1.

\section{Conclusions}

Our data demonstrate a role for STIM1/TRPC1-dependent SOCE in mediating oscillatory patterns of spontaneous and 
netrin-1-potentiated filopodial $\mathrm{Ca}^{2+}$ entries that underlie axonal growth cone guidance both in vitro and in vivo.

\section{Methods}

Molecular constructs

Xenopus STIM1 (XSTIM1) [GenBank: BC126011] was identified by BLAST searches of the GenBank database using human STIM1 cDNA sequence. The coding region of XSTIM1 gene was isolated by RT-PCR, sequenced and cloned into the pCS2 vector (gift of D. Turner, University of Michigan). The following constructs of XSTIM1 and its mutants were generated by site-directed mutagenesis (Strategene) or by PCR based on previous studies of mammalian STIM1 mutants [16]: YFP-XSTIM1-WT, YFP-XSTIM1-DN, YFP-XSTIM1-CA, and mCherry-XS TIM1-DN (Figure 2A). Different XSTIM constructs were in vitro transcribed with the MMESSAGE MMACHINE SP6 kit (Ambion). pN1-Lck-GCaMP3 plasmid was obtained from Addgene (plasmid \#26974, [25]), cloned into pCS2 vector using BamHI and XbaI sites and in vitro transcribed with mMESSAGE mMACHINE SP6 kit (Ambion).

\section{Xenopus embryo injection and spinal neuron culture}

Blastomere injections of mRNAs or morpholinos into early stages of Xenopus embryos and culturing of spinal neurons from these injected embryos were performed as previously described $[20,23,29,56]$. Briefly, fertilized embryos were injected at the two- or four-cell stage with mRNA (2-3 ng/embryo). A control morpholinos or morpholinos specific for Xenopus TRPC1 (XTRPC1-MO) was previously described [20]. Uninjected or injected embryos at stage 22 were used for cultures of spinal neurons as previous described [20,23]. All the procedures involving Xenopus frogs and embryos were carried out in accordance to the NIH guideline for animal use and have been approved by the Institutional Animal Care and Use Committee (IACUC) of Emory University.

\section{RT-PCR and Whole-mount in situ hybridization of Xenopus embryos}

Neural tube and notochord were isolated from the dorsal section of the stage 25-26 Хenopus embryos after dissection with microsurgical scissors and incubation with collagenase (type I, Sigma). Total RNA was prepared by using TRIzol Reagent (invitrogen) and treated with the RNasefree DNAse I (Roche) to remove genomic DNA. The extracted RNA was reverse transcribed by using M-MLV reverse transcriptase (Invitrogen) and random hexamers (Roche). PCR amplification was performed using Taq polymerase (Fermentas). The -RT lane is the negative control of the RT-PCR on neural tube tissue RNA in the absence of a reverese transcriptase. The PCR primers are as follows; XSTIM1-forward, 5' CCAGAACCTTGGAA
GAGGTG 3', XSTIM1-reverse, 5' GACTGAATGGTAC CGGCTGT 3'; XODC-forward, 5' CAGCTAGCTGTG GTGTGG 3', XODC-rev, 5' CAACATGGAAACTCACACC 3'. For whole-mount in situ hybridization, the digoxigenin (DIG)-UTP-labelled antisense RNA was used as previously described $[23,57]$. The C-terminal region of XSTIM1 corresponding to amino acid 192-668 was used for the specific anti-sense and sense probes. The labelled probe was detected with alkaline phosphatase-conjugated anti-DIG antibody (Fab fragments) and visualized with the BM purple AP substrate (Roche Applied Science). Selected embryos from whole-mount in situ hybridization were embedded in a sucrose and Tissue-Tek O.C.T medium, completely frozen and cross-sectioned at $40 \mu \mathrm{m}$ with a cryostat (Leica CM1850).

\section{Immunocytochemistry}

Xenopus spinal neuron cultures were fixed in 4\% paraformaldehyde in a cacodylate buffer $(0.1 \mathrm{M}$ sodium cacodylate, $0.1 \mathrm{M}$ sucrose, $\mathrm{pH}$ 7.4) for 30 minutes and permeabilized with Triton X-100 (0.1\%) for 10 minutes. The cells were incubated with a rabbit polyclonal antibody against full length human STIM1 (MyBioScource) at a dilution of 1:50 after blocking with 5\% goat serum and labelled with Alexa Fluor 546 goat anti-rabbit secondary (Invitrogen). Fluorescent imaging was captured on an inverted microscope (Nikon Eclipse Ti-E).

\section{Growth cone turning assay}

Microscopic gradients of netrin- $1(5 \mu \mathrm{g} / \mathrm{ml}$ in the pipette) were produced as previously described [29,56,58,59]. Xenopus spinal neurons derived from injected blastomeres were identified under fluorescent microscope and used for turning assay at the room temperature 14 to $20 \mathrm{hrs}$ after plating as previously described $[20,23,29,56]$. The culture was plated on glass coverslip without any coating. The turning angle was defined by the angle between the original direction of neurite extension and a straight line connecting the positions of the center of the growth cone at the onset and the end of the $30 \mathrm{~min}$ period. The rates of neurite extension were calculated based on the net neurite extension during the turning assay. Only those growth cones of isolated neurons with a net neurite extension $>5 \mu \mathrm{m}$ over the 30 -min period were included for analysis. Statistical significance was assessed using the Bootstrap-test.

\section{$\mathrm{Ca}^{2+}$ imaging of cultured Xenopus spinal neurons}

$\mathrm{Ca}^{2+}$ imaging of cultured growth cones of Xenopus spinal neurons was performed as previously described $[23,29,56]$. Specifically, isolated Xenopus spinal neurons were cultured on glass coverslip without coating, loaded with Fluo-4 AM (2 $\mu \mathrm{M}$, Molecular Probes) for 30 minutes, rinsed with the Modified Ringer used for growth cone 
turning assay, and imaged after bath-application of netrin$1(10 \mathrm{ng} / \mathrm{ml})$. For store-operated $\mathrm{Ca}^{2+}$ entry experiment, neurons were bathed in $\mathrm{Ca}^{2+}$-free media with CPA $(25 \mu \mathrm{M})$ to deplete intracellular $\mathrm{Ca}^{2+}$ stores, and imaged after re-addition of extracellular $\mathrm{Ca}^{2+}(1.5 \mathrm{mM})$. Growth cones expressing mCherry-XSTIM1-DN proteins were identified under fluorescent microscope and selected for further $\mathrm{Ca}^{2+}$ imaging. Imaging was carried out using a Zeiss 510 META system equipped with a 20X objective (NA 0.8). Excitation was at $488 \mathrm{~nm}$ by argon laser and the emitted fluorescence was collected at 500-560 nm. Fluorescence and bright-field images were simultaneously acquired at every 30 seconds with a frame scan. The mean fluorescence intensity of each time point was measured over a fixed circular region of interest that covers the entire growth cone and normalized to the average fluorescence intensity that was measured during a 2 minutes baseline period (prior to the netrin-1 application or addition of $1.5 \mathrm{mM} \mathrm{Ca}^{2+}$ solution).

For filopodial $\mathrm{Ca}^{2+}$ imaging, Lck-GCaMP3 mRNA was injected into early staged embryos without or with other mRNAs or morpholino. Spinal neurons were cultured on the glass coverslip coated with poly-D-lysine and laminin, which increases the number and length of filopodia [60], in serum-free culture medium. In our netrin-1-induced filopodial $\mathrm{Ca}^{2+}$ entries experiments, the spinal neurons were incubated in MR solution with the addition of cAMP analog Sp-cAMP $(25 \mu \mathrm{M})$ to counterbalance the laminin's effect of reducing cAMP levels in growth cones [61] and mimic the condition of our in vitro turning assay where laminin coating on the glass culture dish was omitted. Live cell imaging of $\mathrm{Ca}^{2+}$ transients was performed on an inverted microscope (Nikon Eclipse Ti-E) equipped with a 60X Apo TIRF objective (NA 1.49), and EMCCD camera (Photometrics) using NIS-Elements software (Nikon). Excitation was at $488 \mathrm{~nm}$ and the emitted fluorescence was collected at $520 \mathrm{~nm}$ and Lck-GCaMP3 fluorescence images were acquired at every 200 milliseconds. To determine several characteristics of filopodial $\mathrm{Ca}^{2+}$ entries, including the incidence, frequency and initiation sites of transients, the Kymographs (spatio-temporal map) were created from the images of the user-defined segmented line one pixel in width spanning the filopodium from the time-lapse movies with NIH ImageJ software. Grayscale values for this linear region of interest (ROI) for each frame of the time series were transformed into pseudocolored images to show time-dependent changes in intracellular $\mathrm{Ca}^{2+}$ concentration $\left(\left[\mathrm{Ca}^{2+}\right]_{\mathrm{i}}\right)$ along the length of the ROI (y-axis) over time ( $\mathrm{x}$-axis).

\section{Immunoprecipitation and immunoblotting}

For co-immunoprecipitation, protein lysates were prepared from the Xenopus brain explants including spinal cords dissected from the embryos at stage 26-28 using lysis buffer containing 1\% Triton X-100, $150 \mathrm{mM} \mathrm{NaCl}$, $10 \mathrm{mM}$ Tris-Cl (pH 7.4), $1 \mathrm{mM}$ EDTA, $1 \mathrm{mM}$ EGTA, 0.5\% Nonidet P-40, $0.2 \mathrm{mM}$ Na-orthovanadate, and protease inhibitor cocktail. They were incubated with the appropriate antibody for 3-4 hours at $4^{\circ} \mathrm{C}$, followed by incubation with protein $\mathrm{A} / \mathrm{G}$ agarose beads (Pierce) for overnight at $4^{\circ} \mathrm{C}$. Mouse anti-c-Myc monoclonal antibody (Roche Applied Science) and rabbit anti-GFP polyclonal antibody (Abcam) were used for immunoprecipitation and immunoblotting.

\section{Whole-mount immunocytochemistry}

Embryos at stage 25-26 were fixed and processed for immunocytochemistry as previously described [20,23,62]. Monoclonal antibody 3A10, specific for commissural interneurons $[23,63]$, was obtained from the Developmental Studies Hybridoma Bank at the University of Iowa and used at a dilution of 1:100. Secondary antibodies were used at a dilution of 1:250. Confocal images of sagittal views of embryos were taken with a Zeiss LSM 510 META system and Z-series reconstructions were processed with the Zeiss LSM image acquisition program as previously described [23]. Statistical significance was assessed using the Bootstrap-test.

\section{Additional files}

\begin{abstract}
Additional file 1: Figure S1. Alignment of STIM1 amino acid sequences of Xenopus laevis, Xenopus tropicalis and human. Identical amino acid residues are highlighted in dark. Dashes indicate gaps inserted for maximal alignment score. The red box indicates the amino acid (D65, within the EF hand motif) that was mutated in the dominant negative form of XSTIM1.
\end{abstract}

Additional file 2: Figure S2. Association of XSTIM1 with TRPC1. Shown are sample westernblots for co-immunoprecipitation of human TRPC1 (myc-hTRPC1-WT) and wild-type (YFP-XSTIM1-WT) or constitutively active STIM1 (YFP-XSTIM1-CA) expressed in Xenopus embryonic neural tissues.

Additional file 3: Movie 1. Filopodial $\mathrm{Ca}^{2+}$ entries are generated by SOCE. A pseudocolored Lck-GCaMP3 fluorescent $\mathrm{Ca}^{2+}$ images of Xenopus neuronal growth cones were captured at 200 milliseconds intervals over $7 \mathrm{~min}$ period of the store-depletion and re-addition of extracellular $\mathrm{Ca}^{2+}$ as shown in Figure 4A.

Additional file 4: Movie 2. SOCE-induced filopodial $\mathrm{Ca}^{2+}$ entries are abolished by inhibition of XSTIM1 with XSTIM1-DN overexpression. A pseudocolored Lck-GCaMP3 fluorescent $\mathrm{Ca}^{2+}$ images of Xenopus neuronal growth cones expressing XSTIM1-DN were captured at 200 milliseconds intervals over $7 \mathrm{~min}$ period of the store-depletion and re-addition of extracellular $\mathrm{Ca}^{2+}$.

Additional file 5: Movie 3. SOCE-induced filopodial $\mathrm{Ca}^{2+}$ entries are attenuated by inhibition of XTRPC1 with XTRPC 1-MO overexpression. A pseudocolored Lck-GCaMP3 fluorescent $\mathrm{Ca}^{2+}$ images of Xenopus neuronal growth cones expressing XTRPC1-MO were captured at 200 milliseconds intervals over $7 \mathrm{~min}$ period of the store-depletion and re-addition of extracellular $\mathrm{Ca}^{2+}$

Additional file 6: Movie 4. Oscillatory spontaneous filopodial $\mathrm{Ca}^{2+}$ entries. A pseudocolored Lck-GCaMP3 fluorescent $\mathrm{Ca}^{2+}$ images of Xenopus neuronal growth cones were captured at 200 milliseconds intervals over $7 \mathrm{~min}$ period in Modified Ringer solutions ( $1 \mathrm{mM}$ extracellular $\mathrm{Ca}^{2+}$ ) as shown in Figure 5A. 
Additional file 7: Movie 5. Spontaneous filopodial $\mathrm{Ca}^{2+}$ entries are abolished by inhibition of XSTIM1 with XSTIM1-DN overexpression. A pseudocolored Lck-GCaMP3 fluorescent $\mathrm{Ca}^{2+}$ images of Xenopus neuronal growth cones expressing XSTIM1-DN were captured at 200 milliseconds intervals over 7 min period in Modified Ringer solutions.

Additional file 8: Movie 6. Spontaneous filopodial $\mathrm{Ca}^{2+}$ entries are abolished by inhibition of XTRPC1 with XTRPC1-MO overexpression. A pseudocolored Lck-GCaMP3 fluorescent $\mathrm{Ca}^{2+}$ images of Xenopus neuronal growth cones expressing XTRPC1-MO were captured at 200 milliseconds intervals over 7 min period in Modified Ringer solutions.

Additional file 9: Movie 7. Netrin-1-potentiated filopodial $\mathrm{Ca}^{2+}$ entries. A pseudocolored Lck-GCaMP3 fluorescent $\mathrm{Ca}^{2+}$ images of Xenopus neuronal growth cone were captured at 200 milliseconds intervals in Modified Ringer solution (1 mM extracellular $\mathrm{Ca}^{2+}$ ) with Sp-cAMP $(25 \mu \mathrm{M})$ before and after bath application of netrin-1 $(10 \mathrm{ng} / \mathrm{ml})$ as shown in Figure 4D.

Additional file 10: Movie 8. Dynamic translocation of STIM1 into neuronal filopodia in response to store-depletion. Time-lapse fluorescent images of Xenopus neuronal growth cone expressing YFP-XSTIM1 before $\left(1 \mathrm{mM} \mathrm{Ca}^{2+}\right)$ and after store $\mathrm{Ca}^{2+}$ depletion $\left(0 \mathrm{mM} \mathrm{Ca}^{2+} / \mathrm{CPA}\right)$. Images were captured at 2 seconds intervals.

\section{Competing interests}

The authors declare that they have no competing interests.

\section{Authors' contributions}

SS designed and performed all the experiments, and wrote the manuscript. JQZ oversaw the project, designed some of the experiments, and revised the manuscript with SS. GLM provided guidance to the project and contributed to the writing of the manuscript. All authors read and approved the final manuscript.

\section{Acknowledgements}

We thank P.F. Worley for help and discussion. This work was supported in part by grants from National Institutes of Health to JQZ (GM083889 and GM084363) and to GLM. (NS048271 and HD069184).

\section{Author details}

'Departments of Cell Biology and Neurology, Emory University School of Medicine, Atlanta, GA 30078, USA. ${ }^{2}$ Center for Neurodegenerative Diseases, Emory University School of Medicine, Atlanta, GA 30322, USA. ${ }^{3}$ Institute for Cell Engineering, Johns Hopkins University School of Medicine, Baltimore, MD 21205, USA. ${ }^{4}$ Department of Neurology, Johns Hopkins University School of Medicine, Baltimore, MD 21205, USA. ${ }^{5}$ The Solomon H. Snyder Department of Neuroscience, Johns Hopkins University School of Medicine, Baltimore, MD 21205, USA.

Received: 5 November 2013 Accepted: 23 November 2013 Published: 1 December 2013

\section{References}

1. Zheng JQ, Poo MM: Calcium signaling in neuronal motility. Annu Rev Cell Dev Biol 2007, 23:375-404.

2. Cahalan MD: STIMulating store-operated Ca(2+) entry. Nat Cell Biol 2009, 11:669-677.

3. Lewis RS: Store-operated calcium channels: new perspectives on mechanism and function. Cold Spring Harb Perspect Biol 2011, 3(12). doi: 10.1101/cshperspect.a003970.

4. Kachoei BA, Knox RJ, Uthuza D, Levy S, Kaczmarek LK, Magoski NS: A store-operated $\mathrm{Ca}(2+)$ influx pathway in the bag cell neurons of Aplysia. J Neurophysiol 2006, 96:2688-2698.

5. Li D, Herault K, Oheim M, Ropert N: FM dyes enter via a store-operated calcium channel and modify calcium signaling of cultured astrocytes. Proc Natl Acad Sci U S A 2009, 106:21960-21965.

6. Gemes G, Bangaru ML, Wu HE, Tang Q, Weihrauch D, Koopmeiners AS, Cruikshank JM, Kwok WM, Hogan QH: Store-operated Ca ${ }^{2+}$ entry in sensory neurons: functional role and the effect of painful nerve injury. J Neurosci 2011, 31:3536-3549.
7. Venkiteswaran $\mathrm{G}$, Hasan $\mathrm{G}$ : Intracellular $\mathrm{Ca}^{2+}$ signaling and store-operated $\mathrm{Ca}^{2+}$ entry are required in Drosophila neurons for flight. Proc Natl Acad Sci U S A 2009, 106:10326-10331.

8. Tang F, Kalil K: Netrin-1 induces axon branching in developing cortical neurons by frequency-dependent calcium signaling pathways. J Neurosci 2005, 25:6702-6715.

9. Baba A, Yasui T, Fujisawa S, Yamada RX, Yamada MK, Nishiyama N, Matsuki $\mathrm{N}$, Ikegaya Y: Activity-evoked capacitative $\mathrm{Ca}^{2+}$ entry: implications in synaptic plasticity. J Neurosci 2003, 23:7737-7741.

10. Steinbeck JA, Henke N, Opatz J, Gruszczynska-Biegala J, Schneider L, Theiss S, Hamacher N, Steinfarz B, Golz S, Brustle O, et al: Store-operated calcium entry modulates neuronal network activity in a model of chronic epilepsy. Exp Neurol 2011, 232:185-194.

11. Geraldo S, Gordon-Weeks PR: Cytoskeletal dynamics in growth-cone steering. J Cell Sci 2009, 122:3595-3604.

12. Zheng JQ, Wan JJ, Poo MM: Essential role of filopodia in chemotropic turning of nerve growth cone induced by a glutamate gradient. J Neurosci 1996, 16:1140-1149.

13. Davenport RW, Dou P, Rehder V, Kater SB: A sensory role for neuronal growth cone filopodia. Nature 1993, 361:721-724.

14. Gomez TM, Robles E, Poo M, Spitzer NC: Filopodial calcium transients promote substrate-dependent growth cone turning. Science 2001, 291:1983-1987.

15. Nicol $X$, Hong KP, Spitzer NC: Spatial and temporal second messenger codes for growth cone turning. Proc Natl Acad Sci U S A 2011, 108:13776-13781.

16. Huang GN, Zeng W, Kim JY, Yuan JP, Han L, Muallem S, Worley PF: STIM1 carboxyl-terminus activates native SOC, I(crac) and TRPC1 channels. Nat Cell Biol 2006, 8:1003-1010.

17. Liu X, Cheng KT, Bandyopadhyay BC, Pani B, Dietrich A, Paria BC, Swaim WD, Beech $D$, Yildrim $E$, Singh $B B$, et al: Attenuation of store-operated $\mathrm{Ca}^{2+}$ current impairs salivary gland fluid secretion in TRPC1(-/-) mice. Proc Natl Acad Sci U S A 2007, 104:17542-17547.

18. Tojima T, Hines JH, Henley JR, Kamiguchi H: Second messengers and membrane trafficking direct and organize growth cone steering. Nature reviews Neuroscience 2011, 12:191-203.

19. Hong K, Nishiyama M, Henley J, Tessier-Lavigne M, Poo M: Calcium signalling in the guidance of nerve growth by netrin-1. Nature 2000, 403:93-98.

20. Shim S, Goh EL, Ge S, Sailor K, Yuan JP, Roderick HL, Bootman MD, Worley PF, Song $H$, Ming GL: XTRPC1-dependent chemotropic guidance of neuronal growth cones. Nat Neurosci 2005, 8:730-735.

21. Wang GX, Poo MM: Requirement of TRPC channels in netrin-1-induced chemotropic turning of nerve growth cones. Nature 2005, 434:898-904.

22. Tojima T: Intracellular signaling and membrane trafficking control bidirectional growth cone guidance. Neurosci Res 2012, 73:269-274.

23. Shim S, Yuan JP, Kim JY, Zeng W, Huang G, Milshteyn A, Kern D, Muallem S, Ming GL, Worley PF: Peptidyl-prolyl isomerase FKBP52 controls chemotropic guidance of neuronal growth cones via regulation of TRPC1 channel opening. Neuron 2009, 64:471-483.

24. Hogan $P G$, Lewis RS, Rao A: Molecular basis of calcium signaling in lymphocytes: STIM and ORAI. Annu Rev Immunol 2010, 28:491-533.

25. Shigetomi E, Kracun S, Khakh BS: Monitoring astrocyte calcium microdomains with improved membrane targeted GCaMP reporters. Neuron Glia Biol 2010, 6:183-191.

26. Kerstein PC, Jacques-Fricke BT, Rengifo J, Mogen BJ, Williams JC, Gottlieb PA, Sachs F, Gomez TM: Mechanosensitive TRPC1 channels promote calpain proteolysis of talin to regulate spinal axon outgrowth. J Neurosci 2013, 33:273-285.

27. Soboloff J, Rothberg BS, Madesh M, Gill DL: STIM proteins: dynamic calcium signal transducers. Nature reviews Molecular cell biology 2012, 13:549-565.

28. Song H, Ming G, He Z, Lehmann M, McKerracher L, Tessier-Lavigne M, Poo $\mathrm{M}$ : Conversion of neuronal growth cone responses from repulsion to attraction by cyclic nucleotides. Science 1998, 281:1515-1518.

29. Ming GL, Song HJ, Berninger B, Holt CE, Tessier-Lavigne M, Poo MM: CAMPdependent growth cone guidance by netrin-1. Neuron 1997, 19:1225-1235.

30. Serafini T, Colamarino SA, Leonardo ED, Wang H, Beddington R, Skarnes WC, Tessier-Lavigne M: Netrin-1 is required for commissural axon guidance in the developing vertebrate nervous system. Cell 1996, 87:1001-1014.

31. Kennedy TE, Serafini T, de la Torre JR, Tessier-Lavigne M: Netrins are diffusible chemotropic factors for commissural axons in the embryonic spinal cord. Cell 1994, 78:425-435. 
32. Mitchell CB, Gasperini RJ, Small DH, Foa L: STIM1 is necessary for store-operated calcium entry in turning growth cones. J Neurochem 2012, 122:1155-1166.

33. Li Y, Jia YC, Cui K, Li N, Zheng ZY, Wang YZ, Yuan XB: Essential role of TRPC channels in the guidance of nerve growth cones by brain-derived neurotrophic factor. Nature 2005, 434:894-898.

34. Ming G, Song H, Berninger B, Inagaki N, Tessier-Lavigne M, Poo M: Phospholipase C-gamma and phosphoinositide 3-kinase mediate cytoplasmic signaling in nerve growth cone guidance. Neuron 1999, 23:139-148.

35. Takei K, Shin RM, Inoue T, Kato K, Mikoshiba K: Regulation of nerve growth mediated by inositol 1,4,5-trisphosphate receptors in growth cones. Science 1998, 282:1705-1708.

36. Akiyama H, Matsu-ura T, Mikoshiba K, Kamiguchi H: Control of neuronal growth cone navigation by asymmetric inositol 1,4,5-trisphosphate signals. Sci Signal 2009, 2:ra34.

37. Xie $Y$, Hong $Y$, Ma $X Y$, Ren XR, Ackerman S, Mei L, Xiong WC: DCC-dependent phospholipase $C$ signaling in netrin-1-induced neurite elongation. J Biol Chem 2006, 281:2605-2611.

38. Xie Y, Ding YQ, Hong Y, Feng Z, Navarre S, Xi CX, Zhu XJ, Wang CL, Ackerman SL, Kozlowski D, et al: Phosphatidylinositol transfer protein-alpha in netrin-1-induced PLC signalling and neurite outgrowth. Nat Cell Biol 2005, 7:1124-1132

39. Yuan JP, Kim MS, Zeng W, Shin DM, Huang G, Worley PF, Muallem S: TRPC channels as STIM1-regulated SOCs. Channels (Austin) 2009, 3:221-225.

40. Yuan JP, Zeng W, Huang GN, Worley PF, Muallem S: STIM1 heteromultimerizes TRPC channels to determine their function as store-operated channels. Nat Cell Biol 2007, 9:636-645.

41. Kim MS, Zeng W, Yuan JP, Shin DM, Worley PF, Muallem S: Native Store-operated $\mathrm{Ca}^{2+}$ Influx Requires the Channel Function of Orai1 and TRPC1. J Biol Chem 2009, 284:9733-9741.

42. Grigoriev I, Gouveia SM, van der Vaart B, Demmers J, Smyth JT, Honnappa S, Splinter D, Steinmetz MO, Putney JW Jr, Hoogenraad CC, Akhmanova A: STIM1 is a MT-plus-end-tracking protein involved in remodeling of the ER. Curr Biol 2008, 18:177-182.

43. Lefkimmiatis K, Srikanthan M, Maiellaro I, Moyer MP, Curci S, Hofer AM: Store-operated cyclic AMP signalling mediated by STIM1. Nat Cell Biol 2009, 11:433-442.

44. Cheng KT, Liu X, Ong HL, Swaim W, Ambudkar IS: Local Ca(2) + entry via Orai1 regulates plasma membrane recruitment of TRPC1 and controls cytosolic $\mathrm{Ca}(2)+$ signals required for specific cell functions. PLOS Biol 2011, 9:e1001025.

45. Ong HL, Cheng KT, Liu X, Bandyopadhyay BC, Paria BC, Soboloff J, Pani B, Gwack Y, Srikanth S, Singh BB, et al: Dynamic assembly of TRPC1-STIM1-Orai1 ternary complex is involved in store-operated calcium influx. Evidence for similarities in store-operated and calcium release-activated calcium channel components. J Biol Chem 2007, 282:9105-9116.

46. Shigetomi E, Tong X, Kwan KY, Corey DP, Khakh BS: TRPA1 channels regulate astrocyte resting calcium and inhibitory synapse efficacy through GAT-3. Nat Neurosci 2012, 15:70-80.

47. Shekarabi M, Kennedy TE: The netrin-1 receptor DCC promotes filopodia formation and cell spreading by activating Cdc42 and Rac1. Mol Cell Neurosci 2002, 19:1-17.

48. Robles E, Woo S, Gomez TM: Src-dependent tyrosine phosphorylation at the tips of growth cone filopodia promotes extension. J Neurosci 2005, 25:7669-7681.

49. Han J, Han L, Tiwari P, Wen Z, Zheng JQ: Spatial targeting of type II protein kinase $A$ to filopodia mediates the regulation of growth cone guidance by cAMP. J Cell Biochem Supp/ 2007, 176:101-111.

50. Mattila PK, Lappalainen P: Filopodia: molecular architecture and cellular functions. Nature reviews Molecular cell biology 2008, 9:446-454.

51. Dent EW, Gupton SL, Gertler FB: The growth cone cytoskeleton in axon outgrowth and guidance. Cold Spring Harb Perspect Biol 2011, 3(3). doi: 10.1101/cshperspect.a001800

52. Gomez TM, Snow DM, Letourneau PC: Characterization of spontaneous calcium transients in nerve growth cones and their effect on growth cone migration. Neuron 1995, 14:1233-1246.

53. Wedel B, Boyles RR, Putney JW Jr, Bird GS: Role of the store-operated calcium entry proteins Stim 1 and Orai1 in muscarinic cholinergic receptor-stimulated calcium oscillations in human embryonic kidney cells.

J Physiol 2007, 579:679-689.
54. Bird GS, Hwang SY, Smyth JT, Fukushima M, Boyles RR, Putney JW Jr: 1STIM1 is a calcium sensor specialized for digital signaling. Curr Biol 2009, 19:1724-1729.

55. Di Capite J, Ng SW, Parekh AB: Decoding of cytoplasmic $\mathrm{Ca}(2+)$ oscillations through the spatial signature drives gene expression. Curr Biol 2009, 19:853-858.

56. Ming GL, Wong ST, Henley J, Yuan XB, Song HJ, Spitzer NC, Poo MM: Adaptation in the chemotactic guidance of nerve growth cones. Nature 2002, 417:411-418

57. Harland RM: In situ hybridization: an improved whole-mount method for Xenopus embryos. Methods Cell Biol 1991, 36:685-695.

58. Lohof AM, Quillan M, Dan Y, Poo MM: Asymmetric modulation of cytosolic CAMP activity induces growth cone turning. J Neurosci 1992, 12:1253-1261.

59. Zheng JQ, Felder M, Connor JA, Poo MM: Turning of nerve growth cones induced by neurotransmitters. Nature 1994, 368:140-144.

60. Rivas RJ, Burmeister DW, Goldberg DJ: Rapid effects of laminin on the growth cone. Neuron 1992, 8:107-115.

61. Hopker VH, Shewan D, Tessier-Lavigne M, Poo M, Holt C: Growth-cone attraction to netrin-1 is converted to repulsion by laminin-1. Nature 1999, 401:69-73.

62. Gomez TM, Spitzer NC: In vivo regulation of axon extension and pathfinding by growth-cone calcium transients. Nature 1999, 397:350-355.

63. Phelps PE, Alijani A, Tran TS: Ventrally located commissural neurons express the GABAergic phenotype in developing rat spinal cord. J Comp Neurol 1999, 409:285-298.

doi:10.1186/1756-6606-6-51

Cite this article as: Shim et al:: A critical role for STIM1 in filopodial calcium entry and axon guidance. Molecular Brain 2013 6:51.

\section{Submit your next manuscript to BioMed Central and take full advantage of:}

- Convenient online submission

- Thorough peer review

- No space constraints or color figure charges

- Immediate publication on acceptance

- Inclusion in PubMed, CAS, Scopus and Google Scholar

- Research which is freely available for redistribution 